\title{
CONTROL OF THE BILINEAR INDICATOR CUBE TESTING PROPERTY
}

\author{
Eric T. Sawyer and Ignacio Uriarte-Tuero
}

McMaster University, Department of Mathematics and Statistics 1280 Main Street West, Hamilton, Ontario, Canada L8S 4K1; sawyer@mcmaster.ca

Michigan State University, Department of Mathematics

619 Red Cedar Road, East Lansing, MI 48824, U.S.A.; ignacio@math.msu.edu

Abstract. We show that the $\alpha$-fractional bilinear indicator/cube testing constant

$$
\mathcal{B I C} \mathcal{T}_{T^{\alpha}}(\sigma, \omega) \equiv \sup _{Q \in \mathcal{P}^{n}} \sup _{E, F \subset Q} \frac{1}{\sqrt{|Q|_{\sigma}|Q|_{\omega}}}\left|\int_{F} T_{\sigma}^{\alpha}\left(\mathbf{1}_{E}\right) \omega\right|,
$$

defined for any $\alpha$-fractional singular integral $T^{\alpha}$ on $\mathbf{R}^{n}$ with $0<\alpha<n$, is controlled by the classical $\alpha$-fractional Muckenhoupt constant $A_{2}^{\alpha}(\sigma, \omega)$, provided the product measure $\sigma \times \omega$ is diagonally reverse doubling (in particular if it is reverse doubling) with exponent exceeding $2(n-\alpha)$.

Moreover, this control is sharp within the class of diagonally reverse doubling product measures. In fact, every product measure $\mu \times \mu$, where $\mu$ is an Ahlfors-David regular measure $\mu$ with exponent $n-\alpha$, has diagonal exponent $2(n-\alpha)$ and satisfies $A_{2}^{\alpha}(\mu, \mu)<\infty$ and $\mathcal{B I C T}_{I^{\alpha}}(\mu, \mu)=\infty$, which has implications for the $L^{2}$ trace inequality of the fractional integral $I^{\alpha}$ on domains with fractional boundary.

When combined with the main results in arXiv:1906.05602, 1907.07571 and 1907.10734, the above control of $\mathcal{B I C T}_{T^{\alpha}}$ for $\alpha>0$ yields a $T 1$ theorem for doubling weights with appropriate diagonal reverse doubling, i.e. the norm inequality for $T^{\alpha}$ is controlled by cube testing constants and the $\alpha$-fractional one-tailed Muckenhoupt constants $\mathcal{A}_{2}^{\alpha}$ (without any energy assumptions), and also yields a corresponding cancellation condition theorem for the kernel of $T^{\alpha}$, both of which hold for arbitrary $\alpha$-fractional Calderón-Zygmund operators $T^{\alpha}$.

We do not know if the analogous result for $\mathcal{B I C T}_{H}(\sigma, \omega)$ holds for the Hilbert transform $H$ in case $\alpha=0$, but we show that $\mathcal{B I C}_{H^{\mathrm{dy}}}(\sigma, \omega)$ is not controlled by the Muckenhoupt condition $\mathcal{A}_{2}^{\alpha}(\omega, \sigma)$ for the dyadic Hilbert transform $H^{\text {dy }}$ and doubling weights $\sigma, \omega$.

\section{Introduction}

We give precise statements of our main results in Subsection 1.4 below, but first we recall the definitions of doubling, reverse doubling, Muckenhoupt conditions and Poisson integrals; then the notion of weighted norm inequality for a standard singular integral, and the associated testing conditions; and finally the bilinear indicator/cube testing theorem from [Sa1], [Sa2] and [Sa3].

1.1. Definitions. Denote by $\mathcal{P}^{n}$ the collection of cubes in $\mathbf{R}^{n}$ having sides parallel to the coordinate axes. A positive locally finite Borel measure $\mu$ on $\mathbf{R}^{n}$ is said to satisfy the doubling condition if there is a pair of constants $(\beta, \gamma) \in(0,1)^{2}$,

https://doi.org/10.5186/aasfm.2021.4664

2020 Mathematics Subject Classification: Primary 42B20.

Key words: Hilbert transform, T1 theorem, two weights, Muckenhoupt conditions, doubling weights, reverse doubling weights, energy conditions, bilinear indicator testing, Bellman function.

E. Sawyer has been partially supported by an NSERC grant.

I. Uriarte-Tuero has been partially supported by grant MTM2015-65792-P (MINECO, Spain). 
called doubling parameters, such that with $|Q|_{\mu}=\mu(Q)$,

$$
|\beta Q|_{\mu} \geq \gamma|Q|_{\mu}, \quad \text { for all cubes } Q \in \mathcal{P}^{n},
$$

and the reverse doubling condition if there is a pair of constants $(\beta, \gamma) \in(0,1)^{2}$, called reverse doubling parameters, such that

$$
|\beta Q|_{\mu} \leq \gamma|Q|_{\mu}, \quad \text { for all cubes } Q \in \mathcal{P}^{n} .
$$

Note that the inequality in (1.2) has been reversed from that in the definition of the doubling condition in (1.1).

A familiar equivalent reformulation of (1.1) is that there is a positive constant $C_{\text {doub }}$, called the doubling constant, and a positive constant $C$, such that $|2 Q|_{\mu} \leq$ $C_{\text {doub }}|Q|_{\mu}$ for all cubes $Q \in \mathcal{P}^{n}$. More important for us is yet another characterization that follows by iterating (1.1): $\mu$ is doubling if and only if there exists a positive constant $\theta_{\mu}^{\text {doub }}$, called a doubling exponent, such that

$$
\sup _{Q \in \mathcal{P}^{n}} \frac{|t Q|_{\mu}}{|Q|_{\mu}} \leq t^{\theta_{\mu}^{\text {doub }}}, \quad \text { for all sufficiently large } t<\infty .
$$

Similarly there is the analogous reformulation of (1.2): $\mu$ is reverse doubling if and only if there exists a positive constant $\theta_{\mu}^{\text {rev }}$, called a reverse doubling exponent, and a positive constant $C$, such that

$$
\sup _{Q \in \mathcal{P}^{n}} \frac{|s Q|_{\mu}}{|Q|_{\mu}} \leq s^{\theta_{\mu}^{\text {rev }}}, \quad \text { for all sufficiently small } s>0 .
$$

A doubling exponent $\theta_{\mu}^{\text {doub }}$ of a doubling measure $\mu$ is necessarily large, namely $\theta_{\mu}^{\text {doub }} \geq n$, and a reverse doubling exponent $\theta_{\mu}^{\text {rev }}$ of a reverse doubling measure $\mu$ is necessarily small, namely $\theta_{\mu}^{\text {rev }} \leq n$, with Lebesgue measure satisfying the extreme case $\theta_{d x}^{\text {rev }}=n=\theta_{d x}^{\text {doub }}$. Indeed, with $\Omega_{N} \equiv\left\{\alpha \in \mathbf{N}^{n}: 0 \leq \alpha_{i} \leq N-1\right\}$, we have for $k$ large,

$$
\begin{aligned}
3^{k n}\left|3^{k} Q\right|_{\mu} & \leq \sum_{\alpha \in \Omega_{3} k}\left|3^{k+1}(Q+\ell(Q) \alpha)\right|_{\mu} \leq \sum_{\alpha \in \Omega_{3^{k}}} 3^{(k+1) \theta_{\mu}^{\text {doub }}}|Q+\ell(Q) \alpha|_{\mu} \\
& \leq 3^{(k+1) \theta_{\mu}^{\text {doub }}}\left|3^{k} Q\right|_{\mu},
\end{aligned}
$$

which implies $\theta_{\mu}^{\text {doub }} \geq n$. Similarly $\theta_{\mu}^{\text {rev }} \leq n$.

Finally it is well known that doubling implies reverse doubling. Indeed, assuming $t \geq 5$ in the definition of $\theta_{\mu}^{\text {doub }}$, we obtain for any cube $Q$ in a dyadic grid $\mathcal{D}$,

$$
\begin{aligned}
|3 Q \backslash Q|_{\mu} & =\sum_{I \in \mathcal{D}: I \subset 3 Q \backslash Q, \ell(I)=\ell(Q)}|I|_{\mu} \\
& \geq \sum_{I \in \mathcal{D}: I \subset 3 Q \backslash Q, \ell(I)=\ell(Q)} 5^{-\theta_{\mu}^{\text {doub }}}|5 I|_{\mu} \geq\left(3^{n}-1\right) 5^{-\theta_{\mu}^{\text {doub }}}|Q|_{\mu} \\
& \Longrightarrow|Q|_{\mu}=|3 Q|_{\mu}-|3 Q \backslash Q|_{\mu} \leq\left(1-\frac{3^{n}-1}{5^{\theta_{\mu}^{\text {doub }}}}\right)|3 Q|_{\mu},
\end{aligned}
$$

with a similar inequality for larger $t$. The converse fails since in particular, reverse doubling measures can vanish on open sets, see Example 7 below, while doubling measures cannot.

Let $\sigma$ and $\omega$ be locally finite positive Borel measures on $\mathbf{R}^{n}$, and denote by $\mathcal{P}^{n}$ the collection of all cubes in $\mathbf{R}^{n}$ with sides parallel to the coordinate axes. For $0 \leq \alpha<n$, 
the classical $\alpha$-fractional Muckenhoupt condition for the weight pair $(\sigma, \omega)$ is given by

$$
A_{2}^{\alpha}(\sigma, \omega) \equiv \sup _{Q \in \mathcal{P}^{n}} \frac{|Q|_{\sigma}}{|Q|^{1-\frac{\alpha}{n}}} \frac{|Q|_{\omega}}{|Q|^{1-\frac{\alpha}{n}}}<\infty
$$

and the corresponding one-tailed condition by

$$
\mathcal{A}_{2}^{\alpha}(\sigma, \omega) \equiv \sup _{Q \in \mathcal{Q}^{n}} \mathcal{P}^{\alpha}(Q, \sigma) \frac{|Q|_{\omega}}{|Q|^{1-\frac{\alpha}{n}}}<\infty,
$$

where the reproducing Poisson integral $\mathcal{P}^{\alpha}$ is given by

$$
\mathcal{P}^{\alpha}(Q, \mu) \equiv \int_{\mathbf{R}^{n}}\left(\frac{|Q|^{\frac{1}{n}}}{\left(|Q|^{\frac{1}{n}}+\left|x-x_{Q}\right|\right)^{2}}\right)^{n-\alpha} d \mu(x) .
$$

1.2. Standard fractional singular integrals, the norm inequality and testing conditions. Let $0 \leq \alpha<n$ and $\kappa_{1}, \kappa_{2} \in \mathbf{N}$. We define a standard $\left(\kappa_{1}+\delta, \kappa_{2}+\delta\right)$-smooth $\alpha$-fractional CZ kernel $K^{\alpha}(x, y)$ to be a function $K^{\alpha}: \mathbf{R}^{n} \times$ $\mathbf{R}^{n} \rightarrow \mathbf{R}$ satisfying the following fractional size and smoothness conditions for some $\delta>0$ : For $x \neq y$, and with $\nabla_{1}$ denoting gradient in the first variable, and $\nabla_{2}$ denoting gradient in the second variable,

$$
\left|\nabla_{1}^{j} K^{\alpha}(x, y)\right| \leq C_{C Z}|x-y|^{\alpha-j-n}, \quad 0 \leq j \leq \kappa_{1},
$$

$$
\left|\nabla_{1}^{\kappa_{1}} K^{\alpha}(x, y)-\nabla_{1}^{\kappa_{1}} K^{\alpha}\left(x^{\prime}, y\right)\right| \leq C_{C Z}\left(\frac{\left|x-x^{\prime}\right|}{|x-y|}\right)^{\delta}|x-y|^{\alpha-\kappa_{1}-n}, \quad \frac{\left|x-x^{\prime}\right|}{|x-y|} \leq \frac{1}{2},
$$

and where the same inequalities hold for the adjoint kernel $K^{\alpha, *}(x, y) \equiv K^{\alpha}(y, x)$, in which $x$ and $y$ are interchanged, and where $\kappa_{1}$ is replaced by $\kappa_{2}$, and $\nabla_{1}$ by $\nabla_{2}$.

If $T^{\alpha}$ is the $\alpha$-fractional singular integral operator associated with the CZ kernel $K^{\alpha}$, then the norm constant $\mathfrak{N}_{T^{\alpha}}=\mathfrak{N}_{T^{\alpha}}(\sigma, \omega)$ is the least constant in the two weight norm inequality

$$
\left(\int_{\mathbf{R}^{n}}\left|T^{\alpha}(f \sigma)\right|^{2} d \omega\right)^{\frac{1}{2}} \leq \mathfrak{N}_{T^{\alpha}}(\sigma, \omega)\left(\int_{\mathbf{R}^{n}}|f|^{2} d \sigma\right)^{\frac{1}{2}}
$$

taken over all suitable truncations, see e.g. [SSU].

The cube testing conditions associated with an $\alpha$-fractional singular integral operator $T^{\alpha}$ introduced in [SSU] are given by

$$
\begin{aligned}
\left(\mathfrak{T}_{T^{\alpha}}(\sigma, \omega)\right)^{2} & \equiv \sup _{Q \in \mathcal{P}^{n}} \frac{1}{|Q|_{\sigma}} \int_{Q}\left|T_{\sigma}^{\alpha} \mathbf{1}_{Q}\right|^{2} \omega<\infty, \\
\left(\mathfrak{T}_{\left(T^{\alpha}\right)^{*}}(\omega, \sigma)\right)^{2} & \equiv \sup _{Q \in \mathcal{P}^{n}} \frac{1}{|Q|_{\omega}} \int_{Q}\left|\left(T_{\sigma}^{\alpha}\right)^{*} \mathbf{1}_{Q}\right|^{2} \sigma<\infty,
\end{aligned}
$$

1.3. The $\mathcal{B I C} \mathcal{T}$ theorem. The bilinear indicator/cube testing property is

$$
\mathcal{B I C T}_{T^{\alpha}}(\sigma, \omega) \equiv \sup _{Q \in \mathcal{P}^{n}} \sup _{E, F \subset Q} \frac{1}{\sqrt{|Q|_{\sigma}|Q|_{\omega}}}\left|\int_{F} T_{\sigma}^{\alpha}\left(\mathbf{1}_{E}\right) \omega\right|<\infty,
$$

where the second supremum is taken over all compact sets $E$ and $F$ contained in a cube $Q$. In [Sa1], [Sa2] and [Sa3] it is shown that for doubling weights, the cube testing conditions, the one-tailed Muckenhoupt conditions, and the bilinear indicator/cube testing property are sufficient for the norm inequality of an $\alpha$-fractional CZ 
operator. In that theorem, the kernel must satisfy smoothness conditions related to the order of vanishing moments of the weighted Alpert wavelets used (see [RSW]), which in turn depend on the doubling exponents of the weights.

Theorem 1. [Sa1, Sa2, Sa3] Suppose that $\sigma$ and $\omega$ are locally finite positive doubling Borel measures on $\mathbf{R}^{n}$. Let $0 \leq \alpha<n$. Suppose also that $T^{\alpha}$ is a standard $\left(\kappa_{1}+\delta, \kappa_{2}+\delta\right)$-smooth $\alpha$-fractional Calderón-Zygmund singular integral in $\mathbf{R}^{n}$, where $\kappa_{1}>\theta_{\sigma}^{\text {doub }}$ and $\kappa_{2}>\theta_{\omega}^{\text {doub }}$ exceed the doubling exponents of $\sigma$ and $\omega$. In the case $\alpha=0$, we also assume that $T^{0}$ is bounded on unweighted $L^{2}\left(\mathbf{R}^{n}\right)$. Then

$$
\mathfrak{N}_{T^{\alpha}}(\sigma, \omega) \lesssim \mathfrak{T}_{T^{\alpha}}(\sigma, \omega)+\mathfrak{T}_{T^{\alpha, *}}(\omega, \sigma)+\mathcal{A}_{2}^{\alpha}(\sigma, \omega)+\mathcal{A}_{2}^{\alpha}(\omega, \sigma)+\mathcal{B I C}_{T^{\alpha}}(\sigma, \omega),
$$

where the implied constant depends only $\alpha, n$, and the doubling constants of the measures. Moreover, if in addition one of the measures is an $A_{\infty}$ weight (and if $T^{0}$ is also bounded on unweighted $L^{2}\left(\mathbf{R}^{n}\right)$ in the case $\left.\alpha=0\right)$, then the bilinear indicator/cube testing property can be dropped:

$$
\mathfrak{N}_{T^{\alpha}}(\sigma, \omega) \lesssim \mathfrak{T}_{T^{\alpha}}(\sigma, \omega)+\mathfrak{T}_{T^{\alpha, *}}(\omega, \sigma)+\mathcal{A}_{2}^{\alpha}(\sigma, \omega)+\mathcal{A}_{2}^{\alpha}(\omega, \sigma) .
$$

This theorem raises the following problem.

Problem 2. Suppose that $\sigma$ and $\omega$ are locally finite positive doubling Borel measures on $\mathbf{R}^{n}$. Let $0 \leq \alpha<n$. Suppose also that $T^{\alpha}$ is a standard $\alpha$-fractional Calderón-Zygmund singular integral in $\mathbf{R}^{n}$. Is the two weight bilinear indicator cube testing constant $\mathcal{B I C}_{T^{\alpha}}(\sigma, \omega)$ then controlled by the cube testing constants $\mathfrak{T}_{T^{\alpha}}(\sigma, \omega), \mathfrak{T}_{T^{\alpha, *}}(\omega, \sigma)$ and the one-tailed Muckenhoupt constants $\mathcal{A}_{2}^{\alpha}(\sigma, \omega), \mathcal{A}_{2}^{\alpha}(\omega$, $\sigma)$ ? More generally, is it true that for every $0<\varepsilon<1$,

$$
\mathcal{B I C T}_{T^{\alpha}}(\sigma, \omega) \lesssim \mathfrak{T}_{T^{\alpha}}(\sigma, \omega)+\mathfrak{T}_{T^{\alpha, *}}(\omega, \sigma)+\mathcal{A}_{2}^{\alpha}(\sigma, \omega)+\mathcal{A}_{2}^{\alpha}(\omega, \sigma)+\sqrt{\varepsilon} \mathfrak{N}_{T^{\alpha}}(\sigma, \omega) ?
$$

1.4. Main results. In the next section we will give a positive answer to Problem 2 for $\alpha>0$ and for certain pairs of doubling measures, without assuming one of them is an $A_{\infty}$ weight. Instead, we assume that the product measure $\sigma \times \omega$ is diagonally reverse doubling, with a bound on a diagonal reverse doubling exponent $\theta_{\sigma \times \omega}^{\mathrm{diag}}$, where by definition $\theta_{\sigma \times \omega}^{\mathrm{diag}}$ satisfies

$$
\sup _{Q \in \mathcal{P}^{n}} \frac{|s(Q \times Q)|_{\sigma \times \omega}}{|Q \times Q|_{\sigma \times \omega}}=\sup _{Q \in \mathcal{P}^{n}} \frac{|s Q|_{\sigma}|s Q|_{\omega}}{|Q|_{\sigma}|Q|_{\omega}} \leq s^{\theta_{\sigma \times \omega}^{\mathrm{diag}}}, \quad \text { for all sufficiently small } s>0 .
$$

Remark 3. If $\sigma$ and $\omega$ are reverse doubling with reverse doubling exponents $\theta_{1}$ and $\theta_{2}$ respectively, then the product measure $\sigma \times \omega$ is reverse doubling with reverse doubling exponent $\theta_{1}+\theta_{2}$, hence $\sigma \times \omega$ is diagonally reverse doubling with exponent $\theta_{\sigma \times \omega}^{\text {diag }} \geq \theta_{1}+\theta_{2}$. In particular, if just one of the measures is reverse doubling, then the product measure is diagonally reverse doubling with at least half the exponent.

Actually we prove a bit more, namely that the two weight bilinear indicator cube testing constant $\mathcal{B I C T}_{I^{\alpha}}(\sigma, \omega)$ for the fractional integral operator $I^{\alpha}$ is controlled by the classical Muckenhoupt constant $A_{2}^{\alpha}(\sigma, \omega)$ alone in this case. Note that when $\alpha>0$, we have $\left|T^{\alpha} \nu\right| \leq C I^{\alpha} \nu$ for any positive measure $\nu$. See the next section for more detail.

Theorem 4. Suppose $\sigma$ and $\omega$ are locally finite positive Borel measures on $\mathbf{R}^{n}$, and that the product measure $\sigma \times \omega$ is diagonally reverse doubling with a diagonal reverse doubling exponent $\theta_{\sigma \times \omega}^{\mathrm{diag}}$. Set $\theta=\frac{\theta_{\sigma \times \omega}^{\mathrm{diag}}}{2}$. If $0<\alpha<n<\theta+\alpha$, then with a 
constant $C=C_{\theta, \alpha, n}$ depending only on $\theta, \alpha$, and $n$, we have

$$
\int_{Q} I^{\alpha}\left(\mathbf{1}_{Q} \sigma\right) d \omega \leq C_{\theta, \alpha, n} \sqrt{A_{2}^{\alpha}(\sigma, \omega)} \sqrt{|Q|_{\sigma}|Q|_{\omega}}, \quad \text { for all cubes } Q \in \mathcal{P}^{n} .
$$

Using Ahlfors-David regular measures, we show that the inequality $n<\theta+\alpha$ in Theorem 4 is sharp. As a corollary of Theorems 1 and 4, we obtain a $T 1$ theorem for arbitrary $\alpha$-fractional Calderón-Zygmund operators in this setting. Note that Theorem 1 requires a degree of smoothness for the kernel that is related to the doubling exponents, as opposed to the reverse doubling exponents.

Corollary 5. Suppose that $\sigma$ and $\omega$ are locally finite positive doubling Borel measures on $\mathbf{R}^{n}$, and that the product measure $\sigma \times \omega$ is diagonally reverse doubling with a diagonal reverse doubling exponent $\theta_{\sigma \times \omega}^{\text {diag }}$ and set $\theta=\frac{\theta_{\sigma \times \omega}^{\text {diag }}}{2}$. Suppose $0<\alpha<$ $n<\theta+\alpha$ and that $T^{\alpha}$ is a $\left(\kappa_{1}+\delta, \kappa_{2}+\delta\right)$-smooth standard $\alpha$-fractional CalderónZygmund singular integral in $\mathbf{R}^{n}$ with $\kappa_{1}>\theta_{\sigma}^{\text {doub }}$ and $\kappa_{2}>\theta_{\omega}^{\text {doub }}$. Then

$$
\mathfrak{N}_{T^{\alpha}}(\sigma, \omega) \lesssim \mathfrak{T}_{T^{\alpha}}(\sigma, \omega)+\mathfrak{T}_{T^{\alpha, *}} \omega(, \sigma)+\mathcal{A}_{2}^{\alpha}(\sigma, \omega)+\mathcal{A}_{2}^{\alpha}(\omega, \sigma),
$$

where the implied constant depends on $\alpha, n$, and the doubling constants for $\sigma$ and $\omega$. Moreover, in terms of cancellation conditions on the kernel $K^{\alpha}(x, y)$ of $T^{\alpha}$, we have

$$
\mathfrak{N}_{T^{\alpha}}(\sigma, \omega) \lesssim \mathfrak{A}_{K^{\alpha}}(\sigma, \omega)+\mathfrak{A}_{K^{\alpha, *}}(\omega, \sigma)+\mathcal{A}_{2}^{\alpha}(\sigma, \omega)+\mathcal{A}_{2}^{\alpha}(\omega, \sigma),
$$

where $\mathfrak{A}_{K^{\alpha}}(\sigma, \omega)$ and $\mathfrak{A}_{K^{\alpha, *}}(\omega, \sigma)$ denote the least positive constants so that

$$
\int_{\left|x-x_{0}\right|<N}\left|\int_{\varepsilon<|x-y|<N} K^{\alpha}(x, y) d \sigma(y)\right|^{2} d \omega(x) \leq \mathfrak{A}_{K^{\alpha}}(\sigma, \omega) \int_{\left|x_{0}-y\right|<N} d \sigma(y),
$$

for all $0<\varepsilon<N$ and $x_{0} \in \mathbf{R}^{n}$,

along with a similar inequality with constant $\mathfrak{A}_{K^{\alpha, *}}(\omega, \sigma)$, in which the measures $\sigma$ and $\omega$ are interchanged and $K^{\alpha}(x, y)$ is replaced by $K^{\alpha, *}(x, y)=K^{\alpha}(y, x)$.

In the third section, we will adapt Nazarov's construction from [NV] to give a negative answer to the analogous question for the dyadic Hilbert transform $H^{\text {dy }}$ (a particular martingale transform) in Theorem 4, namely that $H^{\text {dy }}$, which is of course bounded on unweighted $L^{2}(\mathbf{R})$, can fail the inequality

$$
\left|\int_{Q} H^{\mathrm{dy}}\left(\mathbf{1}_{Q} \sigma\right) d \omega\right| \leq C \sqrt{|Q|_{\sigma}|Q|_{\omega}}, \quad \text { for all intervals } Q .
$$

for all positive constants $C$, no matter the doubling constants of $\sigma$ and $\omega$. Let $\mathcal{D}^{0}$ denote the set of dyadic intervals contained in the unit interval $[0,1]$, and let $H^{\text {dy }}$ denote the dyadic Hilbert transform

$$
H^{\mathrm{dy}} \mu(x) \equiv \frac{1}{2} \sum_{I \in \mathcal{D}^{0}: x \in I} \triangle_{I} \mu, \quad \triangle_{I} \mu \equiv\left(E_{I_{-}} \mu-E_{I_{+}} \mu\right), \quad E_{I} \mu \equiv \frac{1}{|I|} \int_{I} d \mu,
$$

where $I_{-}$and $I_{+}$are the left and right hand dyadic children of $I$. Note that $H^{\mathrm{dy}} \mu(x)=\sum_{I \in \mathcal{D}^{0}}\left\langle\mu, h_{I}\right\rangle \frac{1}{\sqrt{|I|}} \mathbf{1}_{I}$ where $\left\{h_{I}\right\}_{I \in \mathcal{D}^{0}}$ is the Haar basis of $L_{0}^{2}([0,1]) \equiv$ $\left\{f \in L^{2}(0,1): \int_{0}^{1} f=0\right\}$, and where of course $\mu(x)=\sum_{I \in \mathcal{D}^{0}}\left\langle\mu, h_{I}\right\rangle h_{I}$ for $\mu \in$ $L_{0}^{2}(0,1)$. 
Theorem 6. (Adaptation of [NV]) For every $\Gamma>1$ and $\tau>0$ sufficiently small, there exist positive weights $u$ and $v$ on the unit interval $[0,1]$ satisfying

$$
\begin{aligned}
& \int_{0}^{1} H^{\mathrm{dy}} v(x) u(x) d x \geq \Gamma \sqrt{\left(\int_{0}^{1} u(x) d x\right)\left(\int_{0}^{1} v(x) d x\right)}, \\
& \left(\frac{1}{|I|} \int_{I} u(x) d x\right)\left(\frac{1}{|I|} \int_{I} v(x) d x\right) \leq 1, \quad \text { for all } I \in \mathcal{D}^{0}, \\
& 1-\tau<\frac{E_{I_{-}} u}{E_{I_{+}} u}, \frac{E_{I_{-}} v}{E_{I_{+}} v}<1+\tau, \quad \text { for all } I \in \mathcal{D}^{0} .
\end{aligned}
$$

From the second line we obtain the two-tailed Muckenhoupt condition $\mathcal{A}_{2}(u, v) \leq$ $C$ for $\tau>0$ sufficiently small, independent of $\Gamma$, and from the third line, we obtain the doubling conditions for $u$ and $v$ with doubling constants arbitrarily close to 2 for $\tau>0$ sufficiently small, independent of $\Gamma$. See $[N V]$ for the routine proofs of these latter assertions.

Finally, in the appendix we discuss one of the main reasons for restricting our attention to pairs of doubling weights here, and complete the optimal range for a certain parameter in a characterization of doubling in [Sa1].

\section{Bilinear cube testing for $\alpha>0$}

For $\alpha>0$ we use the domination $T^{\alpha} f \leq C I^{\alpha}|f|$ to obtain

$$
\left|\int_{F} T^{\alpha}\left(\mathbf{1}_{E} \sigma\right) d \omega\right| \leq C \int_{F} I^{\alpha}\left(\mathbf{1}_{E} \sigma\right) d \omega \leq C \int_{Q} I^{\alpha}\left(\mathbf{1}_{Q} \sigma\right) d \omega, \quad E, F \subset Q .
$$

Let $\mathfrak{B} \mathfrak{C T}_{I^{\alpha}}(\sigma, \omega)$ denote the best constant in the bilinear cube testing inequality for the fractional integral $I^{\alpha}$,

$$
\int_{Q} I^{\alpha}\left(\mathbf{1}_{Q} \sigma\right) d \omega \leq \mathfrak{B C} \mathfrak{T}_{I^{\alpha}}(\sigma, \omega) \sqrt{|Q|_{\sigma}|Q|_{\omega}}, \quad \text { for all cubes } Q \in \mathcal{P}^{n} .
$$

The constant $\mathfrak{B} \mathfrak{C T}_{I^{\alpha}}(\sigma, \omega)$ is at most the restricted weak type norm constant $\mathfrak{R W T}_{I^{\alpha}}(\sigma, \omega)$ of $I^{\alpha}: L^{2,1}(\sigma) \rightarrow L^{2, \infty}(\omega)$ (which by duality is the same for the inequality $\left.I^{\alpha}: L^{2,1}(\omega) \rightarrow L^{2, \infty}(\sigma)\right)$, but a characterization of the restricted weak type constant $\mathfrak{R W T}_{I^{\alpha}}(\sigma, \omega)$ has yet to be found. Indeed, the restricted weak type constant $\mathfrak{R W T}_{I^{\alpha}}(\sigma, \omega)$ for $I^{\alpha}$ is the smallest constant satisfying

$\int I^{\alpha}(f \sigma) g d \omega \leq \mathfrak{R W T} I_{I^{\alpha}}(\sigma, \omega)\|f\|_{L^{2,1}(\sigma)}\|g\|_{L^{2, \infty}(\omega)}$, for all $f \in L^{2,1}(\sigma), g \in L^{2, \infty}(\omega)$,

which is in turn equivalent to

$\int_{F} I^{\alpha}\left(\mathbf{1}_{E} \sigma\right) d \omega \leq \mathfrak{R W T}_{I^{\alpha}}(\sigma, \omega) \sqrt{|E|_{\sigma}|F|_{\omega}}, \quad$ for all compact subsets $E, F \subset \mathbf{R}^{n}$,

by results in Stein and Weiss $[\mathrm{SW}]$. Then setting $E=F=Q$ yields (2.1) with $\mathfrak{B C} \mathfrak{T}_{I^{\alpha}}(\sigma, \omega) \leq \mathfrak{R W T}_{I^{\alpha}}(\sigma, \omega)$.

Unfortunately, there is no known simple $e^{1}$ characterization of the harmless looking testing inequality (2.1), and in fact the only known simple sufficient condition for (2.1) to hold is that $A_{2}^{\alpha}(\sigma, \omega)<\infty$ and one of the measures is an $A_{\infty}$ weight, see [Sa2]. Since we are assuming $A_{2}^{\alpha}(\sigma, \omega)<\infty$ in all of our work above anyways, and since $A_{2}^{\alpha}(\sigma, \omega)<\infty$ is necessary for $(2.1)$ to hold, we now consider the problem of

\footnotetext{
${ }^{1}$ By simple characterization, we mean using conditions of Muckenhoupt type.
} 
characterizing those weight pairs for which $\mathfrak{B} \mathfrak{C T}_{I^{\alpha}}(\sigma, \omega)$ is controlled by $A_{2}^{\alpha}(\sigma, \omega)$, i.e. there is a positive constant $C$ satisfying

$$
\int_{Q} I^{\alpha}\left(\mathbf{1}_{Q} \sigma\right) d \omega \leq C \sqrt{A_{2}^{\alpha}(\sigma, \omega)} \sqrt{|Q|_{\sigma}|Q|_{\omega}}, \quad \text { for all cubes } Q \in \mathcal{P}^{n} .
$$

Again, there does not appear to be a simple characterization of (2.2) either, with the only sufficient condition being that mentioned above, namely that one of the measures is an $A_{\infty}$ weight. Theorem 4 above provides a different sufficient condition that involves a diagonal reverse doubling exponent of the product measure $\sigma \times \omega$.

\subsection{Proof of the diagonal reverse doubling Theorem 4.}

Proof. We estimate the left hand side of (2.2) by

$$
\begin{aligned}
\int_{Q} I^{\alpha}\left(\mathbf{1}_{Q} \sigma\right) d \omega & =\iint_{Q \times Q}|x-y|^{\alpha-n} d \sigma(x) d \omega(y) \\
& \leq C_{\alpha, n} \iint_{Q \times Q}\left\{\sum_{k=0}^{\infty} \sum_{I \in \mathcal{D}: \ell(I)=2^{-k} \ell(Q)} \ell(I)^{\alpha-n} \mathbf{1}_{3 I \times 3 I}(x, y)\right\} d \sigma(x) d \omega(y) \\
& =C_{\alpha, n} \sum_{k=0}^{\infty} \sum_{\substack{I \in \mathcal{D} \\
\ell(I)=2^{-k} \ell(Q), I \subset Q}}\left[2^{-k} \ell(Q)\right]^{\alpha-n}|(3 I \times 3 I) \cap(Q \times Q)|_{\sigma \times \omega},
\end{aligned}
$$

and then using that the diagonal reverse doubling exponent $2 \theta$ for $\sigma \times \omega$ satisfies $\theta>n-\alpha$, we obtain that for $I \subset Q$ with $\ell(I)=2^{-k} \ell(Q)$ and $k$ large,

$$
\begin{aligned}
\sqrt{|3 I \times 3 I|_{\sigma \times \omega}} & =\sqrt{\left|2^{-k}\left(2^{k} 3 I \times 2^{k} 3 I\right)\right|_{\sigma \times \omega}} \leq 2^{-k \theta} \sqrt{\left|2^{k} 3 I \times 2^{k} 3 I\right|_{\sigma \times \omega}} \\
& \leq 2^{-k \theta} \sqrt{|9 Q \times 9 Q|_{\sigma \times \omega}} .
\end{aligned}
$$

Using this estimate for $k$ large, and the crude estimate

$$
\sqrt{|3 I \times 3 I|_{\sigma \times \omega}} \leq \sqrt{|9 Q \times 9 Q|_{\sigma \times \omega}}
$$

for $k$ small, we obtain

$$
\begin{aligned}
& \int_{Q} I^{\alpha}\left(\mathbf{1}_{Q} \sigma\right) d \omega \\
& \leq C_{\alpha, n} \ell(Q)^{\alpha-n} \sqrt{|9 Q \times 9 Q|_{\sigma \times \omega}} \sum_{k=0}^{\infty} 2^{-k(\alpha-n)} 2^{-k \theta} \\
& \sum_{\substack{I \in \mathcal{D} \\
\ell(I)=2^{-k} \ell(Q), I \subset Q}} \sqrt{|(3 I \times 3 I) \cap(Q \times Q)|_{\sigma \times \omega}} \\
& \leq C_{\alpha, n} \ell(Q)^{\alpha-n} \sqrt{|9 Q|_{\sigma}|9 Q|_{\omega}} \sum_{k=0}^{\infty} 2^{-k(\theta+\alpha-n)} \\
& \cdot\left(\sum_{\substack{I \in \mathcal{D} \\
\ell(I)=2^{-k} \ell(Q), I \subset Q}}|3 I \cap Q|_{\sigma}\right)^{\frac{1}{2}}\left(\sum_{\substack{I \in \mathcal{D} \\
\ell(I)=2^{-k} \ell(Q), I \subset Q}}|3 I \cap Q|_{\omega}\right)^{\frac{1}{2}}
\end{aligned}
$$




$$
\leq C_{\theta, \alpha, n} \ell(9 Q)^{\alpha-n} \sqrt{|9 Q|_{\sigma}|9 Q|_{\omega}} \sqrt{|Q|_{\sigma}|Q|_{\omega}} \leq C_{\theta, \alpha, n} \sqrt{A_{2}^{\alpha}} \sqrt{|Q|_{\sigma}|Q|_{\omega}} .
$$

2.2. Sharpness of the diagonal reverse doubling exponent. Our sharpness examples will be for the equal weight case $\mu=\sigma=\omega$. We now reformulate the equal weight case of inequality (2.2) using the semigroup property $I^{\alpha}=I^{\frac{\alpha}{2}} \circ I^{\frac{\alpha}{2}}$ and $\beta=\frac{\alpha}{2}$. First, by a result of Muckenhoupt and Wheeden [MW], we have

$\int_{Q} I^{\alpha}\left(\mathbf{1}_{Q} \mu\right) d \mu=\int_{Q} I^{\frac{\alpha}{2}} \circ I^{\frac{\alpha}{2}}\left(\mathbf{1}_{Q} \mu\right) d \mu=\int_{\mathbf{R}^{n}} I^{\beta}\left(\mathbf{1}_{Q} \mu\right)(x)^{2} d x \approx \int_{\mathbf{R}^{n}} M^{\beta}\left(\mathbf{1}_{Q} \mu\right)(x)^{2} d x$, where $M^{\beta} \nu(x) \equiv \sup _{x \in Q}|Q|^{\frac{\beta}{n}-1} \int_{Q} d \nu$ is the fractional maximal function. Thus in the equal weight case $\mu=\sigma=\omega,(2.2)$ is equivalent to

$$
\int_{\mathbf{R}^{n}} M^{\beta}\left(\mathbf{1}_{Q} \mu\right)(x)^{2} d x \leq C \sqrt{A_{2}^{\alpha}(\mu, \mu)}|Q|_{\mu}, \quad \text { for all cubes } Q \in \mathcal{P}^{n} .
$$

Example 7. In the case $\mu=\sigma=\omega=d x_{1}$ is the singular measure in the plane $\mathbf{R}^{2}$ given by one-dimensional Lebesgue measure on the real axis, and with $\alpha=1=\frac{n}{2}$, we have that the reverse doubling exponent of $\mu \times \mu$ is 2 , and that the fractional Muckenhoupt constant is finite, yet $\int_{Q} I^{\alpha}\left(\mathbf{1}_{Q} \sigma\right) d \omega=\infty$, showing that (2.3) can fail when $\theta=n-\alpha$. Indeed, it is trivial that $\theta=1=n-\alpha$. For $Q=[0, R] \times[0, R]$ and $\beta=\frac{\alpha}{2}=\frac{1}{2}$, we have

$$
M^{\beta}\left(\mathbf{1}_{Q} \mu\right)\left(x_{1}, x_{2}\right) \approx x_{2}^{2\left(\frac{\beta}{2}-1\right)} x_{2}=x_{2}^{\beta-1}, \quad x=\left(x_{1}, x_{2}\right) \in Q,
$$

and so

$$
\begin{aligned}
\frac{1}{|Q|_{\mu}} \int_{Q} M^{\beta}\left(\mathbf{1}_{Q} \mu\right)\left(x_{1}, x_{2}\right)^{2} d x_{1} d x_{2} & \approx \frac{1}{R} \int_{0}^{R} \int_{0}^{R}\left(x_{2}^{\beta-1}\right)^{2} d x_{1} d x_{2} \\
& =\int_{0}^{R} x_{2}^{2 \beta-2} d x_{2}=\int_{0}^{R} x_{2}^{-1} d x_{2}=\infty
\end{aligned}
$$

while

$$
\sqrt{A_{2}^{\alpha}(\sigma, \omega)} \approx \sup _{Q=[0, R] \times[0, R]}|Q|^{\frac{\alpha}{n}-1} \cdot \int_{Q} d \mu=\sup _{R>0}\left(R^{2}\right)^{\frac{1}{2}-1} \cdot R=1 .
$$

We can extend this sharpness example to general indices $0<\alpha<n$ using AhlforsDavid regular measures. A measure $\mu$ is said to be Ahlfors-David regular of order $\theta$ if

$$
|3 Q|_{\mu} \approx \ell(Q)^{\theta} \text { whenever }|Q|_{\mu}>0 .
$$

Lemma 8. If $\mu$ is any Ahlfors-David regular measure in $\mathbf{R}^{n}$ of order $n-\alpha$ where $0<\alpha<n$, then (2.3) fails with $\beta=\frac{\alpha}{2}$.

Proof. Suppose that $\mu$ is Ahlfors-David regular of order $\theta$. First we note that

$$
\sqrt{A_{2}^{\alpha}(\mu, \mu)} \approx \sup _{Q \in \mathcal{P}^{n}}|Q|^{\frac{\alpha}{n}-1} \int_{Q} d \mu \approx \sup _{Q \in \mathcal{P}^{n}} \ell(Q)^{\alpha-n} \ell(Q)^{\theta}=1,
$$

if $\theta=n-\alpha$. To show that the left side of (2.3) is infinite for the same choice of $\theta$, we proceed in four steps. Let $\mathfrak{C}^{(N)}(Q)$ denote the collection of dyadic subcubes of $Q$ having side length $\ell\left(Q^{\prime}\right)=2^{-N} \ell(Q)$. Throughout the proof, constants implied by $\approx$ and $\lesssim$ depend only on $\alpha, n$ and the Ahlfors-David constants implicit in the definition $(2.4)$. 
Step 1: Let

$$
\Gamma_{N}(Q) \equiv\left\{Q^{\prime} \in \mathfrak{C}^{(N)}(Q):\left|Q^{\prime}\right|_{\mu}>0\right\}, \quad \text { for } Q \in \mathcal{P}^{n} .
$$

Since $\mu$ is Ahlfors-David regular of order $\theta \equiv n-\alpha$, we have for any cube $Q \in \mathcal{P}^{n}$ that both

$$
\begin{aligned}
& \sum_{Q^{\prime} \in \Gamma_{N}(Q)}\left|3 Q^{\prime}\right|_{\mu} \approx \sum_{Q^{\prime} \in \Gamma_{N}(Q)} \ell\left(Q^{\prime}\right)^{\theta}=\# \Gamma_{N}(Q) \cdot 2^{-N \theta} \ell(Q)^{\theta} \\
& \sum_{Q^{\prime} \in \Gamma_{N}(Q)}\left|3 Q^{\prime}\right|_{\mu} \lesssim|3 Q|_{\mu} .
\end{aligned}
$$

Thus we obtain

$$
\# \Gamma_{N}(Q) \cdot 2^{-N \theta} \ell(Q)^{\theta} \lesssim|3 Q|_{\mu} \approx \ell(Q)^{\theta}, \quad \text { if }|Q|_{\mu}>0
$$

and hence

$$
\# \Gamma_{N}(Q) \lesssim 2^{N \theta}, \quad \text { if } Q \in \mathcal{P}^{n}
$$

In particular there is $N=N_{n, \alpha, \theta}$ sufficiently large that $\mathfrak{C}^{(N)}(Q) \backslash \Gamma_{N}(Q) \neq \emptyset$ for all cubes $Q \in \mathcal{P}^{n}$.

Step 2: Fix a cube $Q$ and let $N=N_{n, \alpha, \mu}$ be as in Step 1. Then $\mathfrak{C}^{(N)}(Q) \backslash \Gamma_{N}(Q) \neq$ $\emptyset$ and so there is $Q^{*} \in \mathfrak{C}^{(N)}(Q)$ with $\left|Q^{*}\right|_{\mu}=0$. Since

$$
\inf _{x \in Q} M^{\beta}\left(\mathbf{1}_{Q} \mu\right)(x) \geq \ell(Q)^{\beta-n} \int_{Q} d \mu,
$$

we then have

$$
\int_{Q^{*}} M^{\beta}\left(\mathbf{1}_{Q} \mu\right)(x)^{2} d x \geq \ell(Q)^{\alpha-2 n}\left(\int_{Q} d \mu\right)^{2} \ell\left(Q^{*}\right)^{n} \approx 2^{-N n} \ell(Q)^{\theta+\alpha-n} \int_{Q} d \mu .
$$

Set $\Omega_{1}(Q) \equiv Q^{*}$. Since $\theta+\alpha-n=0$, there is a positive constant $c_{N}$ such that for $Q \in \mathcal{P}^{n}$,

$$
\int_{\Omega_{1}(Q)} M^{\beta}\left(\mathbf{1}_{Q} \mu\right)(x)^{2} d x \geq c_{N} \int_{Q} d \mu
$$

Step 3: Again fix a cube $Q$ and let $N=N_{n, \alpha, \mu}$ be as in Step 1. Let $\Gamma_{N}(Q)=$ $\left\{Q_{k}\right\}_{k=1}^{K}$ where $K \lesssim 2^{N \theta}$ by (2.5). Then we apply Step 2 to the cube $Q_{k}$ to obtain a cube $Q_{k}^{*}$ with $\left|Q_{k}^{*}\right|_{\mu}=0$ and

$$
\int_{Q_{k}^{*}} M^{\beta}\left(\mathbf{1}_{Q_{k}} \mu\right)(x)^{2} d x \geq c_{N} \int_{Q_{k}} d \mu .
$$

Then with $\Omega_{2} \equiv \bigcup_{k=1}^{K} Q_{k}^{*}$, we obtain upon summing in $k$ that

$$
\int_{\Omega_{2}} M^{\beta}\left(\mathbf{1}_{Q} \mu\right)(x)^{2} d x \geq c_{N} \int_{Q} d \mu
$$

Note that $Q_{k}^{*} \subset Q_{k}$ where $\left|Q_{k}\right|_{\mu}>0$, and that $\left|Q^{*}\right|_{\mu}=0$, which shows that $Q_{k}^{*} \cap Q^{*}=$ $\emptyset$ for all $k$, hence $\Omega_{1} \cap \Omega_{2}=\emptyset$. Thus we have that

$$
\int_{\Omega_{1} \cup \Omega_{2}} M^{\beta}\left(\mathbf{1}_{Q} \mu\right)(x)^{2} d x \geq 2 c_{N} \int_{Q} d \mu .
$$


Step 4: Now repeat Step 3 indefinitely to obtain

$$
\int_{\Omega_{1} \cup \Omega_{2} \cup \ldots \cup \Omega_{m}} M^{\beta}\left(\mathbf{1}_{Q} \mu\right)(x)^{2} d x \geq m c_{N} \int_{Q} d \mu, \quad \text { for all } m \geq 1,
$$

which of course shows that

$$
\int_{Q} M^{\beta}\left(\mathbf{1}_{Q} \mu\right)(x)^{2} d x=\infty
$$

Problem 9. The measures $\mu$ in the sharpness examples above are not however doubling, only reverse doubling. This begs the question of whether or not (2.2) can hold for all pairs of doubling measures, a question we leave open.

Finally, Lemma 8 shows the failure of the trace inequality $I^{\alpha}: L^{2} \rightarrow L^{2}(\partial \Omega)$ for a domain $\Omega \subset \mathbf{R}^{n}$ when $\partial \Omega$ is an Ahlfors-David regular set of order $n-\alpha$. For example $I^{\frac{1}{2}}: L^{2} \rightarrow L^{2}(\partial \Omega)$ fails in the plane if $\partial \Omega$ is the Cantor dust fractal-Example 7 is the case when $\partial \Omega$ is a line.

\section{Failure of $\mathfrak{B C} \mathcal{T}$ for the dyadic Hilbert transform}

We do not know if the analogous inequality for the Hilbert transform on the real line, i.e.

$$
\int_{Q}\left|H\left(\mathbf{1}_{Q} \sigma\right)\right| d \omega \leq C \sqrt{A_{2}(\sigma, \omega)} \sqrt{|Q|_{\sigma}|Q|_{\omega}}, \quad \text { for all intervals } Q,
$$

holds, but we can show that the analogous question for the dyadic Hilbert transform is answered in the negative here (no it can fail) using an adaptation of Nazarov's Bellman construction in [NV].

The following bilinear cube testing condition for the Hilbert transform $H$ is of course implied by restricted weak type for $H$ :

$$
\left|\int_{Q} H\left(\mathbf{1}_{Q} \sigma\right) d \omega\right| \leq \mathcal{B C} \mathcal{T}_{H} \sqrt{|Q|_{\sigma}|Q|_{\omega}}, \quad \text { for all intervals } Q .
$$

Unfortunately we are unable to determine if $\mathcal{B C}_{H}<\infty$. Instead, we will prove here Theorem 6 , that shows the discrete dyadic form of the inequality fails, i.e. that the inequality

$$
\left|\int_{Q} H^{\mathrm{dy}}\left(\mathbf{1}_{Q} \sigma\right) d \omega\right| \leq \mathcal{B C} \mathcal{T}_{H^{\mathrm{dy}}} \sqrt{|Q|_{\sigma}|Q|_{\omega}}, \quad \text { for all dyadic intervals } Q \subset[0,1),
$$

fails. In fact, Theorem 6 is an easy consequence of (1.9) and the following simpler variant of a Bellman construction from [NV].

\subsection{The dyadic Bellman construction.}

Lemma 10. Let $0<\tau<1$. Then for every $\Gamma>1$, there exists a pair of weights $(U, V)$ on the unit interval $I^{0} \equiv[0,1]$, and a positive integer $M \in \mathbf{N}$, such that each of the functions $U, V$ is positive on $[0,1]$ and constant ${ }^{2}$ on every interval $K \in \mathcal{D}^{0}$

\footnotetext{
${ }^{2}$ We do not actually need this constant property here since we are unable to apply the 'supervisor' argument from $[\mathrm{NV}]$ to extend the counterexample to the $\alpha$-fractional Riesz transform on the line when $\alpha>0$.
} 
having side length $2^{-M}$, and moreover,

$$
\begin{aligned}
& \sum_{I \in \mathcal{D}^{0}}\left(\triangle_{I} V\right)\left(E_{I} U\right)|I|>\Gamma \sqrt{\left(E_{I^{0}} U\right)\left(E_{I^{0}} V\right)}, \\
& \left(E_{I} U\right)\left(E_{I} V\right) \leq 1, \quad \text { for all } I \in \mathcal{D}^{0}, \\
& 1-\tau<\frac{E_{I_{-}} U}{E_{I_{+}} U}, \frac{E_{I_{-}} V}{E_{I_{+}} V}<1+\tau, \quad \text { for all } I \in \mathcal{D}^{0} .
\end{aligned}
$$

To prove this lemma we use the Bellman function

$$
\mathcal{B}(x) \equiv \sup _{J \in \mathcal{D}^{0}}\left\{\frac{1}{|J|} \sum_{I \in \mathcal{D}^{0}: I \subset J}\left(\triangle_{I} V\right)\left(E_{I} U\right)|I|:(U, V) \in \mathcal{F}_{J ; x}\right\},
$$

for $x=\left(x_{1}, x_{2}\right) \in(0, \infty)^{2}$ with $x_{1} x_{2}<1$, in analogy with that in $[\mathrm{NV}]$, where $\mathcal{F}_{J ; x}$ consists of those pairs $(U, V)$ of positive functions on $J$ such that

$$
E_{J} U=x_{1}, \quad E_{J} V=x_{2},
$$

and $\left(E_{I} U\right)\left(E_{I} V\right)<1, \quad$ for all $I \in \mathcal{D}^{0}$ with $I \subset J$.

Note that the averages of $U$ and $V$ are only fixed to be $x_{1}$ and $x_{2}$ respectively at the interval $J$. Moreover, while it is the case that $\triangle_{I} V$ can be negative, an appropriate switching of children for each parent replaces $\triangle_{I} V$ with $\left|\triangle_{I} V\right|$ while leaving $E_{I} U$ unaffected, and so we also have

$$
\mathcal{B}(x) \equiv \sup _{J \in \mathcal{D}^{0}}\left\{\frac{1}{|J|} \sum_{I \in \mathcal{D}^{0}: I \subset J}\left|\triangle_{I} V\right|\left(E_{I} U\right)|I|:(U, V) \in \mathcal{F}_{J ; x}\right\},
$$

which shows in particular that $\mathcal{B}(x)$ is positive.

The Bellman function $\mathcal{B}(x)$ satisfies the rescaling property,

$$
\frac{1}{|\widehat{J}|} \sum_{I \in \mathcal{D}^{0}: I \subset \widehat{J}}\left|\triangle_{I} \widehat{V}\right|\left(E_{I} \widehat{U}\right)|I|=\frac{1}{|J|} \sum_{I \in \mathcal{D}^{0}: I \subset J}\left|\triangle_{I} V\right|\left(E_{I} U\right)|I|,
$$

where $(\widehat{U}, \widehat{V})=\left(S_{a, b} U, S_{a, b} V\right) \in \mathcal{F}_{J ; x}$ with $S_{a, b} f(z)=f\left(T_{a, b}^{-1} z\right)$ and $T_{a, b} y=a y+b$, and where $\widehat{J}=T_{a, b} J$ with $a>0$ and $b \in \mathbf{R}$. Indeed, the affine map $T_{a, b}$ takes an interval $I$ to an interval $T_{a, b} I$ with $\left|T_{a, b} I\right|=a|I|$, and preserves the dyadic structures within the intervals $I$ and $T_{a, b} I$. Moreover, if $a=2^{k}$ and $b=2^{k} \ell$ for some $k \in \mathbf{Z}$ and $\ell \in \mathbf{N}$, then $I \in \mathcal{D}$ if and only if $T_{a, b} I \in \mathcal{D}$. Note that $S_{a, b}$ takes functions $f$ supported in $I$ to functions $S_{a, b} f$ supported in $T_{a, b} I$, and moreover preserves averages over all dyadic intervals $I$, i.e.

$$
\begin{aligned}
E_{T_{a, b} I}\left(S_{a, b} f\right) & =\frac{1}{\left|T_{a, b} I\right|} \int_{T_{a, b} I} f\left(T_{a, b}^{-1} z\right) d z=\frac{1}{\left|T_{a, b} I\right|} \int_{I} f(y) a d y \\
& =\frac{1}{a|I|} \int_{I} f(y) a d y=E_{I} f,
\end{aligned}
$$


as well as the 'difference averages',

$$
\begin{aligned}
\triangle_{T_{a, b} I}\left(S_{a, b} f\right) & =E_{\left(T_{a, b} I\right)_{-}}\left(S_{a, b} f\right)-E_{\left(T_{a, b} I\right)_{+}}\left(S_{a, b} f\right) \\
& =\frac{1}{\left|\left(T_{a, b} I\right)_{-}\right|} \int_{\left(T_{a, b} I\right)_{-}} S_{a, b} f(z) d z-\frac{1}{\left|\left(T_{a, b} I\right)_{+}\right|} \int_{\left(T_{a, b} I\right)_{+}} S_{a, b} f(z) d z \\
& =\frac{1}{\left|\left(T_{a, b} I\right)_{-}\right|} \int_{\left(T_{a, b} I\right)_{-}} f\left(T_{a, b}^{-1} z\right) d z-\frac{1}{\left|\left(T_{a, b} I\right)_{+}\right|} \int_{\left(T_{a, b} I\right)_{+}} f\left(T_{a, b}^{-1} z\right) d z \\
& =\frac{1}{a\left|I_{-}\right|} \int_{I_{-}} f(y) a d y-\frac{1}{a\left|I_{+}\right|} \int_{I_{+}} f(y) a d y=E_{I_{-}} f-E_{I_{+}} f=\triangle_{I} f .
\end{aligned}
$$

Now fix dyadic intervals $J$ and $\widehat{J}$ in $\mathcal{D}^{0}$. Choose an affine map $T_{a, b}$ with $a=2^{k}$ and $b=2^{k} \ell$, for some $k, \ell \in \mathbf{Z}$, that takes the interval $J$ one-to-one and onto the interval $\widehat{J}=T_{a, b} J$. Define functions $\widehat{U}=S_{a, b} U$ and $\widehat{V}=S_{a, b} V$. Then we have

$$
\begin{aligned}
& \frac{1}{|\widehat{J}|} \sum_{I \in \mathcal{D}^{0}: I \subset \widehat{J}}\left|\triangle_{I} \widehat{V}\right|\left(E_{I} \widehat{U}\right)|I|=\frac{1}{\left|T_{a, b} J\right|} \sum_{I \in \mathcal{D}^{0}: I \subset T_{a, b} J}\left|\triangle_{I}\left(S_{a, b} V\right)\right| E_{I}\left(S_{a, b} U\right)|I| \\
& =\frac{1}{\left|T_{a, b} J\right|} \sum_{I \in \mathcal{D}^{0: I \subset J}}\left|\triangle_{T_{a, b} I}\left(S_{a, b} V\right)\right|\left(E_{T_{a, b} I}\left(S_{a, b} U\right)\right)\left|T_{a, b} I\right| \\
& =\frac{1}{a|J|} \sum_{I \in \mathcal{D}^{0}: I \subset J}\left|\triangle_{I} V\right|\left(E_{I} U\right) a|I|=\frac{1}{|J|} \sum_{I \in \mathcal{D}^{0}: I \subset J}\left|\triangle_{I} V\right|\left(E_{I} U\right)|I|,
\end{aligned}
$$

and also $(\widehat{U}, \widehat{V})=\left(S_{a, b} U, S_{a, b} V\right) \in \mathcal{F}_{\widehat{J} ; x}$ since

$$
E_{\widehat{J}}(\widehat{U})=E_{T_{a, b} J}\left(S_{a, b} U\right)=E_{J} U=x_{1} \text { and } E_{\widehat{J}}(\widehat{V})=E_{T_{a, b} J}\left(S_{a, b} V\right)=E_{J} V=x_{2} \text {. }
$$

Now let

$$
\Omega \equiv\left\{x=\left(x_{1}, x_{2}\right) \in(0, \infty)^{2}: x_{1} x_{2}<1\right\} .
$$

Assuming that $\mathcal{B}(x)<\infty$ for all $x \in \Omega$, we will derive a contradiction from Theorem 11 below, thus concluding that $\mathcal{B}(x)$ must be $\infty$ for some $x \in \Omega$, and so in particular that $\sup _{x \in \Omega} \frac{\mathcal{B}(x)}{\sqrt{x_{1} x_{2}}}=\infty$. In any event, this shows that for any $\Gamma>1$ there is $x \in \Omega, J \in \mathcal{D}^{0}$ and $(U, V) \in \mathcal{F}_{J ; x}$ such that

$$
\frac{1}{|J| \sqrt{\left(E_{J} U\right)\left(E_{J} U\right)}} \sum_{I \in \mathcal{D}^{0}: I \subset J}\left(\triangle_{I} V\right)\left(E_{I} U\right)|I|>\Gamma,
$$

which if $J=I^{0}$, as we may assume, gives

$$
\sum_{I \in \mathcal{D}^{0}}\left|\triangle_{I} V\right|\left(E_{I} U\right)|I|>\Gamma \sqrt{\left(E_{I^{0}} U\right)\left(E_{I^{0}} U\right)}
$$

since $\left|I^{0}\right|=1$. This will complete the proof of Lemma 10 upon restricting the sum of the nonnegative terms $\left|\triangle_{I} V\right|\left(E_{I} U\right)|I|$ for $I \in \mathcal{D}^{0}$ to intervals $I$ of side length at least $2^{-M}$ for a sufficiently large $M \in \mathbf{N}$.

We begin by establishing a very strict concavity property of $\mathcal{B}(x)$ in $\Omega$.

Theorem 11. Assume that $\mathcal{B}(x)<\infty$ for all $x \in \Omega$. If $y=\left(y_{1}, y_{2}\right)$ is such that $x, x+y, x-y \in \Omega$, then

$$
\frac{\mathcal{B}(x+y)+\mathcal{B}(x-y)}{2}+2\left|y_{2}\right| x_{1}-\mathcal{B}(x) \leq 0 .
$$


Proof. Fix an interval $J \in \mathcal{D}^{0}$, which we could of course take to be $I^{0}=[0,1)$. Consider two pairs $\left(U_{+}, V_{+}\right)$and $\left(U_{-}, V_{-}\right)$with corresponding intervals $J_{x+y}$ and $J_{x-y}$ that are ' $\eta$-maximizing' for $x+y$ and $x-y$ respectively with $\eta>0$, by which we mean that

$$
\begin{aligned}
\mathcal{B}(x+y)-\eta< & \frac{1}{\left|J_{x+y}\right|} \sum_{I \in \mathcal{D}^{0}: I \subset J_{x+y}}\left|\triangle_{I} V_{+}\right|\left(E_{I} U_{+}\right)|I|, \\
& \quad \text { for } E_{J_{x+y}} U_{+}=x_{1}+y_{1}, E_{J_{x+y}} V_{+}=x_{2}+y_{2}, \\
\mathcal{B}(x-y)-\eta< & \frac{1}{\left|J_{x-y}\right|} \sum_{I \in \mathcal{D}^{0}: I \subset J_{x-y}}\left|\triangle_{I} V_{-}\right|\left(E_{I} U_{-}\right)|I|, \\
& \quad \text { for } E_{J_{x-y}} U_{-}=x_{1}-y_{1}, E_{J_{x-y}} V_{-}=x_{2}-y_{2} .
\end{aligned}
$$

Moreover, we may assume that all of the weights above are constant on sufficiently small intervals. By rescaling with appropriate maps $T_{a, b}$ and $S_{a, b}$ as in (3.3) above, we may suppose that the dyadic intervals $J_{x+y}, J_{x-y}$ have the form $J_{+}, J_{-}$respectively, where $J$ is the interval fixed at the beginning of the proof, and moreover that $U_{ \pm}, V_{ \pm}$ are supported in $J_{ \pm}$.

Following $[\mathrm{NV}]$ we now construct a pair $(\widetilde{U}, \widetilde{V})$ supported in $J$ satisfying

$$
\widetilde{U} \equiv\left\{\begin{array} { l l } 
{ U _ { + } } & { \text { on } J _ { + } } \\
{ U _ { - } } & { \text { on } J _ { - } } \\
{ 0 } & { \text { on } J ^ { c } }
\end{array} \text { and } \widetilde { V } \equiv \left\{\begin{array}{ll}
V_{+} & \text {on } J_{+} \\
V_{-} & \text {on } J_{-} \\
0 & \text { on } J^{c}
\end{array}\right.\right.
$$

We claim that $(\widetilde{U}, \widetilde{V}) \in \mathcal{F}_{J ; x}$. Indeed,

$$
\begin{aligned}
E_{J} \widetilde{U} & =\frac{1}{|J|} \int_{J} \widetilde{U}(x) d x=\frac{1}{|J|} \int_{J_{+}} U_{+}(x) d x+\frac{1}{|J|} \int_{J_{-}} U_{-}(x) d x \\
& =\frac{1}{2}\left\{\frac{1}{\left|J_{+}\right|} \int_{J_{+}} U_{+}(x) d x+\frac{1}{\left|J_{-}\right|} \int_{J_{-}} U_{-}(x) d x\right\} \\
& =\frac{1}{2}\left\{E_{J_{+}} \widetilde{U}+E_{J_{-}} \widetilde{U}\right\}=\frac{1}{2}\left\{x_{1}+y_{1}+x_{1}-y_{1}\right\}=x_{1},
\end{aligned}
$$

and similarly $E_{J} \widetilde{V}=x_{2}$, and of course then

$$
\left(E_{J} \widetilde{U}\right)\left(E_{J} \widetilde{V}\right)=x_{1} x_{2}<1
$$

Turning next to the strict dyadic subintervals $I$ of $J$ we have for $I \subset J_{+}$,

$$
\begin{array}{ll}
E_{I} \widetilde{U}=E_{I} U_{+}, & \triangle_{I} \widetilde{U}=\triangle_{I} U_{+}, \\
E_{I} \widetilde{V}=E_{I} V_{+}, & \triangle_{I} \widetilde{V}=\triangle_{I} V_{+},
\end{array}
$$

and for $I \subset J_{-}$,

$$
\begin{array}{ll}
E_{I} \widetilde{U}=E_{I} U_{-}, & \triangle_{I} \widetilde{U}=\triangle_{I} U_{-}, \\
E_{I} \widetilde{V}=E_{I} V_{-}, & \triangle_{I} \widetilde{V}=\triangle_{I} V_{-} .
\end{array}
$$

Consequently we obtain

$$
\left(E_{I} \widetilde{U}\right)\left(E_{I} \widetilde{V}\right)<1
$$

which completes the proof of our claim that $(\widetilde{U}, \widetilde{V}) \in \mathcal{F}_{J ; x}$. 
Note that we also have

$$
\triangle_{J} \tilde{V}=E_{J_{-}} \tilde{V}-E_{J_{+}} \tilde{V}=E_{J_{-}} V_{-}-E_{J_{+}} V_{+}=\left[\left(x_{2}-y_{2}\right)-\left(x_{2}+y_{2}\right)\right]=-2 y_{2} .
$$

Then with

$$
\mathcal{L}_{J}(f, g) \equiv \sum_{I \in \mathcal{D}^{0}: I \subset J}\left|\triangle_{I} g\right|\left(E_{I} f\right)|I|, \quad \text { for }(f, g) \in \mathcal{F}_{J ; x}
$$

we have

$$
\begin{aligned}
\mathcal{B}(x) \geq & \frac{1}{|J|} \mathcal{L}_{J}(\widetilde{U}, \widetilde{V})=\left|\triangle_{J} \widetilde{V}\right|\left(E_{J} \widetilde{U}\right)+\frac{1}{|J|} \sum_{I \in \mathcal{D}^{0}: I \subset J_{+}}\left|\triangle_{I} \widetilde{V}\right|\left(E_{I} \widetilde{U}\right)|I| \\
& +\frac{1}{|J|} \sum_{I \in \mathcal{D}^{0}: I \subset J_{-}}\left|\triangle_{I} \widetilde{V}\right|\left(E_{I} \widetilde{U}\right)|I| \\
= & 2\left|y_{2}\right| x_{1}+\frac{1}{2} \frac{1}{\left|J_{+}\right|} \sum_{I \in \mathcal{D}^{0}: I \subset J_{+}}\left|\triangle_{I} V_{+}\right|\left(E_{I} U_{+}\right)|I| \\
& +\frac{1}{2} \frac{1}{\left|J_{-}\right|} \sum_{I \in \mathcal{D}^{0}: I \subset J_{-}}\left|\triangle_{I} V_{-}\right|\left(E_{I} U_{-}\right)|I| \\
> & 2\left|y_{2}\right| x_{1}+\frac{1}{2}\{\mathcal{B}(x+y)-\eta+\mathcal{B}(x-y)-\eta\} \\
= & 2\left|y_{2}\right| x_{1}+\frac{\mathcal{B}(x+y)+\mathcal{B}(x-y)}{2}-\eta .
\end{aligned}
$$

Since $\eta>0$ is arbitrary, this gives

$$
\frac{\mathcal{B}(x+y)+\mathcal{B}(x-y)}{2}+2\left|y_{2}\right| x_{1}-\mathcal{B}(x) \leq 0,
$$

and this completes the proof of Theorem 11.

We may assume that $\mathcal{B}(x)$ is finite everywhere on $\Omega$, since otherwise we are done. Then Theorem 11 shows in particular that $\mathcal{B}(x)$ is concave on $\Omega$, and so by a result of Buseman and Feller [BF] (extended to $\mathbf{R}^{n}$ by Alexandrov [Al]), $\mathcal{B}(x)$ is differentiable to second order for almost every $x \in \Omega$. But if the Bellman function $\mathcal{B}$ is twice differentiable at a fixed $x \in \Omega$, Taylor's formula gives

$$
\begin{aligned}
\mathcal{B}(x \pm y) & =\mathcal{B}(x) \pm(y \cdot \nabla) \mathcal{B}(x)+\frac{1}{2} y^{\operatorname{tr}} \nabla^{2} B(x) y+o\left(|y|^{2}\right), \\
\text { i.e. } \frac{\mathcal{B}(x+y)+\mathcal{B}(x-y)}{2} & =\mathcal{B}(x)+\frac{1}{2} y^{\operatorname{tr}} \nabla^{2} B(x) y+o\left(|y|^{2}\right),
\end{aligned}
$$

for sufficiently small $|y|$, and then the full force of Theorem 11 shows that

$$
\frac{1}{2} y^{\operatorname{tr}} \nabla_{x}^{2} B(x) y+o\left(|y|^{2}\right)+2\left|y_{2}\right| x_{1} \leq 0,
$$

i.e. $2\left|y_{2}\right| x_{1} \leq C|y|^{2}$ for sufficiently small $|y|$,

which is clearly impossible since $x_{1}>0$. This shows that $\mathcal{B}(x)=\infty$ for some $x \in \Omega$ as we claimed just before the statement of Lemma 10.

In order to achieve the doubling property in the third line of the conclusion of Lemma 10, we follow [NV] by fixing $0<\tau<1$ and modifying the above proof as follows. 
(1) Replace $\mathcal{F}_{J ; x}$ with $\mathcal{F}_{J ; x, \tau}$ where $\mathcal{F}_{J ; x, \tau}$ consist of those pairs $(U, V)$ of positive functions on $J$ such that

$$
\begin{aligned}
E_{J} U & =x_{1}, \quad E_{J} V=x_{2}, \\
\frac{\left|\triangle_{I} U\right|}{E_{I} U} & \leq \frac{\tau}{10}, \quad \text { for all dyadic } I \subset J, \\
\frac{\left|\triangle_{I} V\right|}{E_{I} V} & \leq \frac{\tau}{10}, \quad \text { for all dyadic } I \subset J, \\
\text { and }\left(E_{I} U\right)\left(E_{I} V\right) & <1, \quad \text { for all } I \in \mathcal{D}^{0} \text { with } I \subset J .
\end{aligned}
$$

(2) Replace $\mathcal{B}(x)$ with $\mathcal{B}_{\tau}(x)$ where

$$
\mathcal{B}_{\tau}(x) \equiv \sup _{J \in \mathcal{D}^{0}}\left\{\frac{1}{|J|} \sum_{I \in \mathcal{D}^{0}: I \subset J}\left|\triangle_{I} V\right|\left(E_{I} U\right)|I|:(U, V) \in \mathcal{F}_{J ; x, \tau}\right\}, \quad \text { for } x \in \Omega \text {. }
$$

Then we obtain from the above argument that $\mathcal{B}_{\tau}(x)=\infty$ for some $x \in \Omega$. Indeed, the analogue of Theorem 11 is now the following theorem.

Theorem 12. Assume that $\mathcal{B}_{\tau}(x)<\infty$ for all $x \in \Omega$. If $y=\left(y_{1}, y_{2}\right)$ is such that $x, x+y, x-y \in \Omega$ and $\frac{2\left|y_{1}\right|}{x_{1}}, \frac{2\left|y_{2}\right|}{x_{2}} \leq \frac{\tau}{10}$, then

$$
\frac{\mathcal{B}_{\tau}(x+y)+\mathcal{B}_{\tau}(x-y)}{2}+2\left|y_{2}\right| x_{1}-\mathcal{B}_{\tau}(x) \leq 0 \text {. }
$$

The point of assuming $\frac{2\left|y_{1}\right|}{x_{1}}, \frac{2\left|y_{2}\right|}{x_{2}} \leq \frac{\tau}{10}$ in the hypotheses of Theorem 12 is that the weight pair $(\widetilde{U}, \widetilde{V})$ constructed in the proof of Theorem 11 above then satisfies $\frac{\left|\triangle_{I} \widetilde{U}\right|}{E_{I} \widetilde{U}}=\frac{2\left|y_{1}\right|}{x_{1}} \leq \frac{\tau}{10}$ and $\frac{\left|\triangle_{I} \widetilde{U}\right|}{E_{I} \widetilde{U}} \leq \frac{\tau}{10}$ for $I \varsubsetneqq J$, and similarly for $\widetilde{V}$, and so we have $(\widetilde{U}, \widetilde{V}) \in \mathcal{F}_{J ; x, \tau}$. The proof of Theorem 12 now proceeds as in the proof of Theorem 11 . The remainder of the argument is unchanged.

This completes the proof of Lemma 10 since one easily verifies that if $\frac{\left|\triangle_{I} U\right|}{E_{I} U} \leq$ $\frac{\tau}{10}$ for all dyadic $I \in \mathcal{D}^{0}$, then $1-\tau<\frac{E_{I_{-}} U}{E_{I_{+}} U}<1+\tau$, for all $I \in \mathcal{D}^{0}$, and similarly for $V$.

Remark 13. The above argument proves that if $\Omega$ is a domain in $\mathbf{R}^{n}$, and $B: \Omega \rightarrow[0, \infty]$ is twice differentiable at some $x \in \Omega$, then we cannot have

$$
B(x) \geq \frac{B(x+y)+B(x-y)}{2}+2\left|y_{2}\right| x_{1}, \quad \text { for all } y \text { such that } x \pm y \in \Omega .
$$

This simple observation doesn't apply to the Bellman function for testing conditions in $[\mathrm{NV}$, see (3.1)-(3.4)], since in particular, the inequality for the three dimensional Bellman function in [NV, (3.13)] has $y_{2}^{2} x_{1}$ in place of $2\left|y_{2}\right| x_{1}$ :

$$
B(x)+x_{2} \frac{\partial B}{\partial x_{3}} y_{1}^{2} \geq \frac{B(x+y)+B(x-y)}{2}+y_{2}^{2} x_{1} .
$$

Moreover, the two problems are quite different, as the conclusion in [NV, see (4.1)(4.3) plus doubling] yields a Muckenhoupt doubling weight pair that satisfies one testing condition for the dyadic Hilbert transform, but not the other; while Theorem 6 above yields a Muckenhoupt doubling weight pair that cannot satisfy either testing condition, since they each imply bilinear testing.

Problem 14. Is the bilinear cube testing constant $\mathcal{B C}_{H}(\sigma, \omega)$ for the Hilbert transform $H$ controlled by $\mathcal{A}_{2}^{\alpha}(\sigma, \omega)$ when the measures $\sigma, \omega$ are doubling? 


\section{Appendix}

Here we complete the analysis of energy nondegeneracy conditions, introduced in [Sa1], which arise when using Calderón-Zygmund decompositions in connection with weighted Alpert wavelets. We begin by recalling some notation from [Sa1]. We say that a polynomial $P(y)=\sum_{0 \leq|\beta|<\kappa} c_{\beta} y^{\beta}$ of degree less than $\kappa$ is normalized if

$$
\sup _{y \in Q_{0}}|P(y)|=1, \quad \text { where } Q_{0} \equiv \prod_{i=1}^{n}\left[-\frac{1}{2}, \frac{1}{2}\right) .
$$

Definition 15. Denote by $c_{Q}$ the center of the cube $Q$, and by $\ell(Q)$ its side length, and for any polynomial $P$ set

$$
P^{Q}(y) \equiv P\left(c_{Q}+\ell(Q) y\right)
$$

We say that $P(x)$ is $Q$-normalized if $P^{Q}$ is normalized. Denote by $\left(\mathcal{P}_{\kappa}^{Q}\right)_{\text {norm }}$ the set of $Q$-normalized polynomials of degree less than $\kappa$.

Thus a $Q$-normalized polynomial has its supremum norm on $Q$ equal to 1 . Recall from (1.1) that a locally finite positive Borel measure $\mu$ on $\mathbf{R}^{n}$ is doubling if there exist constants $0<\beta, \gamma<1$ such that

$$
|\beta Q|_{\mu} \geq \gamma|Q|_{\mu}, \quad \text { for all cubes } Q \text { in } \mathbf{R}^{n}
$$

Note that $\sup _{y \in Q}|P(y)|=\left\|\mathbf{1}_{Q} P\right\|_{L^{\infty}(\mu)}$ for any cube $Q$, polynomial $P$, and nontrivial doubling measure $\mu$. It was shown in [Sa1] that if $\mu$ is doubling on $\mathbf{R}^{n}$, then for every $\kappa \in \mathbf{N}$ there exists a positive constant $C_{\kappa}$ such that

$$
\begin{aligned}
|Q|_{\mu} \leq & C_{\kappa} \int_{Q}|P(x)|^{2} d \mu(x), \text { for all cubes } Q \text { in } \mathbf{R}^{n}, \\
& \text { and for all } Q \text {-normalized polynomials } P \text { of degree less than } \kappa .
\end{aligned}
$$

It was also shown that conversely, if $\kappa>2 n$, then (4.2) implies that $\mu$ is doubling. Here we extend the converse to the optimal range $\kappa \geq 1$.

Lemma 16. Let $\mu$ be a locally finite positive Borel measure on $\mathbf{R}^{n}$. If (4.2) holds for some positive integer $\kappa \in \mathbf{N}$, then $\mu$ is doubling.

Proof. Assume that (4.2) holds for some $\kappa \in \mathbf{N}$. Momentarily fix a cube $Q$ and an index $1 \leq i \leq n$, and let $a_{Q} \in \mathbf{R}^{n}$ where $Q=\prod_{i=1}^{n}\left[\left(a_{Q}\right)_{i},\left(a_{Q}\right)_{i}+\ell(Q)\right]$. Then the polynomial

$$
P_{i}(x) \equiv \frac{x_{i}-\left(a_{Q}\right)_{i}}{\ell(Q)}
$$

is $Q$-normalized of degree less than $\kappa$, vanishes on the face of the boundary of $Q$ which lies in the hyperplane $\left\{x \in \mathbf{R}^{n}: x_{i}=\left(a_{Q}\right)_{i}\right\}$, and is 1 on the opposite face where $x_{i}=\left(a_{Q}\right)_{i}+\ell(Q)$. Thus for each $0<\varepsilon<1$, there is $\beta<1$, sufficiently close 
to 1 , and independent of the cube $Q$, so that

$$
\begin{aligned}
& |Q|_{\mu} \leq C_{\kappa} \int_{Q}\left|P_{i}\right|^{2} d \mu
\end{aligned}
$$

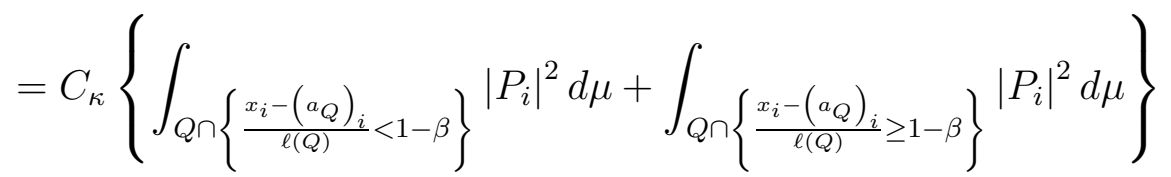

$$
\begin{aligned}
& \leq \varepsilon\left|Q \cap\left\{\frac{x_{i}-\left(a_{Q}\right)_{i}}{\ell(Q)}<1-\beta\right\}\right|_{\mu}+C_{\kappa}\left|Q \cap\left\{\frac{x_{i}-\left(a_{Q}\right)_{i}}{\ell(Q)} \geq 1-\beta\right\}\right|_{\mu} \\
& \leq \varepsilon|Q|_{\mu}+C_{\kappa}\left|Q \cap\left\{\frac{x_{i}-\left(a_{Q}\right)_{i}}{\ell(Q)} \geq 1-\beta\right\}\right|_{\mu} \text {. }
\end{aligned}
$$

Now we focus on the rectangle $Q \cap\left\{\frac{x_{i}-\left(a_{Q}\right)_{i}}{\ell(Q)} \geq 1-\beta\right\}$ that appears on the right hand side above. It can be written as a union of at most $2^{n-1}$ cubes $Q^{\prime} \in \Gamma$ of side length $\beta \ell(Q)$ (thus not necessarily dyadic, and overlapping significantly-e.g. if $Q=$ $[0,1]^{2}$ and $i=1$, then the squares $Q^{\prime}$ are $[1-\beta, 1] \times[0, \beta]$ and $\left.[1-\beta, 1] \times[1-\beta, 1]\right)$, where $\Gamma$ is an index set of size $2^{n-1}$ satisfying

$$
Q \cap\left\{\frac{x_{i}-\left(a_{Q}\right)_{i}}{\ell(Q)} \geq 1-\beta\right\}=\bigcup_{Q^{\prime} \in \Gamma} Q^{\prime} .
$$

Now fix another index $j \neq i$, and for each of these cubes $Q^{\prime}$, apply the above argument with the polynomial $P_{j}$ in place of $P_{i}$. Then we obtain

$$
\begin{aligned}
|Q|_{\mu} \leq & \varepsilon|Q|_{\mu}+C_{\kappa}\left|Q \cap\left\{\frac{x_{i}-\left(a_{Q}\right)_{i}}{\ell(Q)} \geq 1-\beta\right\}\right|_{\mu} \\
\leq & \varepsilon|Q|_{\mu}+C_{\kappa} \sum_{Q^{\prime} \in \Gamma}\left(\varepsilon\left|Q^{\prime}\right|_{\mu}+C_{\kappa}\left|Q^{\prime} \cap\left\{\frac{x_{j}-\left(a_{Q^{\prime}}\right)_{j}}{\ell\left(Q^{\prime}\right)} \geq 1-\beta\right\}\right|_{\mu}\right. \\
\leq & \varepsilon\left(1+2^{n-1} C_{\kappa}\right)|Q|_{\mu} \\
& +2^{n-1} C_{\kappa}^{2} \mid\left. Q \cap\left\{\frac{x_{j}-\left(a_{Q}\right)_{j}}{\beta \ell(Q)} \geq 1-\beta \text { and } \frac{x_{i}-\left(a_{Q}\right)_{i}}{\beta \ell(Q)} \geq 1-\beta\right\}\right|_{\mu},
\end{aligned}
$$

where in the final term we have written $\ell\left(Q^{\prime}\right)=\beta \ell(Q)$ and made the final set bigger by replacing $\left(a_{Q^{\prime}}\right)_{j}$ with the smaller number $\left(a_{Q}\right)_{j}$. By further replacing the second factor of $2^{n-1}$ by its square, we have

$$
\begin{aligned}
|Q|_{\mu} \leq & \varepsilon\left(1+\left[2^{n-1} C_{\kappa}\right]\right)|Q|_{\mu} \\
& +\left[2^{n-1} C_{\kappa}\right]^{2} \mid\left. Q \cap\left\{\frac{x_{j}-\left(a_{Q}\right)_{j}}{\ell(Q)} \geq \beta(1-\beta) \text { and } \frac{x_{i}-\left(a_{Q}\right)_{i}}{\ell(Q)} \geq \beta(1-\beta)\right\}\right|_{\mu}
\end{aligned}
$$

Now we continue this process until we have exhausted the indices in $\{1,2, \ldots, n\}$ and are left with cubes $Q^{\prime}$ that are at distance at least $1-\beta$ from each of the hyperplanes $\left\{x \in \mathbf{R}^{n}: x_{i}=\left(a_{Q}\right)_{i}\right\}$ for $1 \leq i \leq n$.

Then we turn our attention to the remaining $n$ faces of the boundary of $Q$, which lie in the hyperplanes $\left\{x \in \mathbf{R}^{n}: x_{i}=\left(a_{Q}\right)_{i}+\ell(Q)\right\}$ for $1 \leq i \leq n$, using the 
polynomials

We eventually obtain

$$
\widehat{P}_{i}(x) \equiv \frac{\ell(Q)+\left(a_{Q}\right)_{i}-x_{i}}{\ell(Q)}
$$

$|Q|_{\mu} \leq \varepsilon\left(1+\left[2^{n-1} C_{\kappa}\right]+\ldots+\left[2^{n-1} C_{\kappa}\right]^{2 n-1}\right)|Q|_{\mu}+\left[2^{n-1} C_{\kappa}\right]^{2 n}\left|\beta^{2 n-1}(1-\beta) Q\right|_{\mu}$.

Now we choose $\varepsilon=\frac{1}{2\left(1+\left[2^{n-1} C_{\kappa}\right]+\ldots+\left[2^{n-1} C_{\kappa}\right]^{2 n-1}\right)}$ to get

$$
|Q|_{\mu} \leq 2\left[2^{n-1} C_{\kappa}\right]^{2 n}\left|\beta^{2 n-1}(1-\beta) Q\right|_{\mu},
$$

which is (4.1) with $\gamma=\frac{1}{2 C_{\kappa}^{2 n}}$ and $\beta$ replaced by $\beta^{2 n-1}(1-\beta)$.

\section{References}

[Al] Alexandrov, A. D.: Almost everywhere existence of the second differential of a convex function and some properties of convex surfaces connected with it. - Leningrad State Univ. Ann. [Uchenye Zapiski] Math. Ser. 6, 1939, 3-35 (in Russian).

[BF] Busemann, H., and W. Feller: Krümmungseigenschaften konvexer Flächen. - Acta Math. 66, 1936, 1-47.

[MW] Muckenhoupt, B., and R. L. Wheeden: Weighted norm inequalities for fractional integrals. - Trans. Amer. Math. Soc. 192, 1974, 261-274.

[NV] Nazarov, F., and A. VolberG: Bellman function, two-weighted Hilbert transform, and embeddings of the model spaces $K_{\theta}$. - J. Anal. Math. 87, 2002, 385-414.

[RSW] RAhm, R., E. T. SAWyer, and B. D. Wick: Weighted Alpert wavelets. - arXiv: $1808.01223 \mathrm{v} 2$.

[Sa1] SAwYER, E. T.: A Tp theorem with BICT for fractional singular integrals with doubling measures: cancellation conditions for Calderón-Zygmund operators. - arXiv:1906.05602.

[Sa2] SAwYER, E. T.: A restricted weak type inequality with application to a $T p$ theorem and cancellation conditions for CZO's. - arXiv:1907.07571.

[Sa3] Sawyer, E.T.: T1 implies $T p$ polynomial testing: optimal cancellation conditions for CZO's. - arXiv:1907.10734.

[SSU] SAwyer, E.T., C.-Y. Shen, I. URIARTE-Tuero: A two weight theorem for $\alpha$-fractional singular integrals with an energy side condition. - Revista Mat. Iberoam. 32:1, 2016, 79-174.

[SW] Stein, E., and G. Weiss: Fourier analysis on Euclidean spaces. - Princeton Univ. Press, Princeton, New Jersey, 1971.

Received 23 October 2019 • Accepted 11 May 2021 